\title{
Lifetime of merger features of equal-mass disk mergers
}

\author{
Inchan $\mathrm{Ji}^{1}$, Sébastien Peirani ${ }^{2}$, and Sukyoung K. $\mathrm{Yi}^{1}$ \\ 1 Department of Astronomy and Yonsei University Observatory, Yonsei University, 120-749 Seoul, Republic of Korea \\ e-mail: yi@yonsei.ac.kr \\ 2 Institut d'Astrophysique de Paris (UMR 7095: CNRS \& UPMC), 98 bis Bd Arago, 75014 Paris, France
}

Received 29 January 2014 / Accepted 2 May 2014

\section{ABSTRACT}

\begin{abstract}
Context. Detecting post-merger features of merger remnants is highly dependent on the depth of observation images. However, there has not been much discussion of how long the post-merger features are visible under different observational conditions.

Aims. We investigate a merger-feature time useful for understanding the morphological transformation of galaxy mergers via numerical simulations.

Methods. We used $N$-body/hydrodynamic simulations, including gas cooling, star formation, and supernova feedback. We ran a set of simulations with various initial orbital configurations and with progenitor galaxies having different morphological properties mainly for equal-mass mergers. As reference models, we ran additional simulations for non-equal mass mergers and a merger in a large halo potential. Mock images using the SDSS $r$ band were synthesized to estimate a merger-feature time and compare it between the merger simulations.

Results. The mock images suggest that the post-merger features involve a small fraction of stars, and the merger-feature time depends on galaxy interactions. In an isolated environment, the merger-feature time is, on average, $\sim 2$ times the final coalescence time for a shallow surface bright limit of $25 \mathrm{mag} \mathrm{arcsec}^{-2}$. For a deeper surface brightness limit of $28 \mathrm{mag} \operatorname{arcsec}^{-2}$, however, the merger-feature time is a factor of two longer, which is why the detection of post-merger features using shallow surveys has been difficult. Tidal force of a cluster potential is effective in stripping post-merger features out and reduces the merger-feature time.
\end{abstract}

Key words. galaxies: formation - galaxies: interactions - galaxies: evolution - methods: numerical

\section{Introduction}

Since the seminal work of Toomre \& Toomre (1972) illustrating the peculiar features of nearby galaxies, galaxy merger has been considered a fundamental phenomenon in understanding galaxy formation. The Lambda cold dark matter $(\Lambda \mathrm{CDM})$ model predicts the hierarchical galaxy formation which suggests that present-day galaxies are formed from successive accretions and mergers of smaller entities (White \& Rees 1978). In this context, large early-type galaxies are believed to have experienced mergers between disk galaxies in the past (Toomre 1977).

Theoretical perspectives of galaxy interactions help us understand galaxy formation and evolution. In particular, star formation histories and the dynamical properties of galaxy mergers have been investigated through numerical simulations (e.g., Gerhard 1981; Barnes \& Hernquist 1991; Hernquist 1992; Naab $\&$ Burkert 2003; Cox et al. 2006a,b; Di Matteo et al. 2007; Peirani et al. 2010). The numerical simulations suggest that the properties of merger remnants, such as their isophotal shapes, surface density profiles, and the ratio between local rotation to velocity dispersion $(v / \sigma)$, are consistent with those of observed early-type galaxies. In addition, dissipative mergers can account for the central concentration of starbursts between merger remnants (Barnes \& Hernquist 1991; Mihos \& Hernquist 1994; Springel 2000).

However, the gap between observation and theoretical study is still considerable. The properties of galaxies beyond their internal structures, e.g., magnitudes and colors, have rarely been studied primarily because of the difficulty of modeling dust effects in the interstellar medium of galaxies. Various methods have recently been developed to overcome this problem.

One method is the use of Monte Carlo radiative transfers assuming idealized galaxy models or using distributions of gas and stars from numerical simulations (Silva et al. 1998; Jonsson 2006). Although this method provides detailed and extensive panchromatic data for galaxies, it requires high computational costs. The other method is to directly assign spectral data to a single stellar population using population synthesis models. This method is effective because of its simplicity and applicability. Several studies have examined the observable properties of merger remnants by assuming the total amount of dust extinction from empirical data (Kaviraj et al. 2009; Gabor et al. 2011). Therefore, their approaches are well-suited to studying the integrated magnitudes and colors of each galaxy, but not to studying regional differences.

The data provided by large surveys and improvements in numerical modeling give us an opportunity to study all physical processes that occur during the interactions of galaxies. As an illustration, large surveys such as the CfA2 Redshift Survey, the Two-Degree Field (2dF) Galaxy Redshift Survey, the Deep Extragalactic Evolutionary Probe (DEEP), and the Sloan Digital Sky Survey (SDSS) have collected detailed galaxy properties, while hydrodynamic simulations and population synthesis models have been employed to produce the observable properties of merger remnants. Recent studies have tried to compare the photometric quantities derived from numerical simulations to data from large surveys (Springel et al. 2005a; Gabor \& Davé 2012).

Most massive galaxies are expected to undergo a series of merger events (Stewart et al. 2008). It is then necessary to 

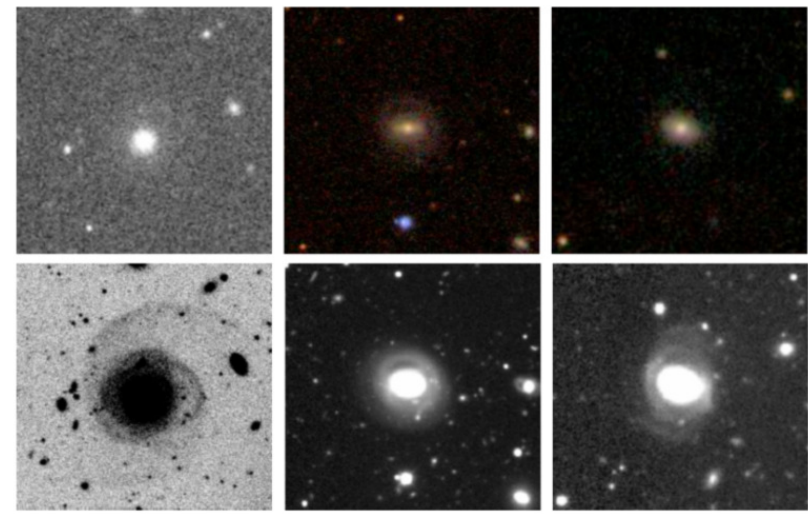

Fig. 1. SDSS images (top) and CTIO $r^{\prime}$ band images (bottom) of galaxies in Abell 2670. The images are courtesy of Yun-Kyeong Sheen. The galaxies in each column are the same object. The surface brightness limits in the SDSS and CTIO observations are $\mu_{\text {limit }} \sim 25$ and $28 \mathrm{mag} \mathrm{arcsec}^{-2}$, respectively. One can see that the deep images reveal faint tidal features around the galaxies.

constrain timescales such as first passage, maximum separation, and final coalescence when we predict the physical properties of galaxies. Observations help identify the timescales using angular separation, line-of-sight radial velocity, and correlation functions (Patton et al. 2000; Barton et al. 2000; Lin et al. 2004; De Propris et al. 2005; Bell et al. 2006; Li et al. 2008). Recent studies have shown that the timescales before the final coalescence can be formulated analytically based on numerical simulations (Boylan-Kolchin et al. 2008; Jiang et al. 2008). However, only a few studies have measured timescales beyond the final coalescence. One such timescale is the merger-feature time, the moment when faint and disturbed features of merger remnants (post-merger features) disappear. Lotz et al. (2008) assumed that the merger-feature time (which they call the "post-merger" stage) ends 1 Gyr after the final coalescence. However, the merger-feature time obviously depends on observational conditions such as surface brightness limits.

Figure 1 shows three early-type galaxies in Abell 2670 observed from the SDSS $\left(\mu_{\text {limit }} \sim 25 \mathrm{mag} \operatorname{arcsec}^{-2}\right)$ and from the CTIO $r^{\prime}$ band $\left(\mu_{\text {limit }}=28 \mathrm{mag} \operatorname{arcsec}^{-2}\right)$ by Sheen et al. (2012). The images from the SDSS show only the central regions of the galaxies and suggest elliptical-like galaxies. However, the deeper images from the CTIO observations clearly show faint structures around the galaxies and thus characterize more accurately the dynamical and relaxation state of the galaxies. Therefore, this suggests that the merger-feature time could be estimated more accurately with the deeper images.

In this work, our aim is to study the merger-feature time after galaxy merger while considering both reasonable dynamical evolutions of galaxy mergers and dust attenuation. We produce a set of merger events using $N$-body/hydrodynamic simulations from which we extract the dust-attenuated spectra of galaxies using both Bruzual \& Charlot (2003) and Calzetti et al. (2000). This modeling allows us to construct mock images of galaxies mimicking observational environments to be compared with observational data.

This paper is organized as follows: the numerical modeling is summarized in Sect. 2, while the methodology used to extract observable properties is described in Sect. 3. In Sect. 4, we compare the merger-feature times of merger remnants. Finally, we discuss the results in Sect. 5.

\section{Numerical modeling}

\subsection{Galaxy modeling and simulations}

The numerical methodology used in this paper is described in detail in Peirani et al. (2009) to which we refer the reader for more information. For the sake of clarity, we summarize the major steps below.

Our study is focused on 1:1 mass-ratio mergers where the final coalescence time is the shortest in the large parameter space (e.g., Boylan-Kolchin et al. 2008). This 1:1 value is also useful for measuring various timescales as we will discuss below. Galaxies are created following the prescription described by Springel et al. (2005b). We use a compound galaxy model consisting of a spherical dark matter halo, a rotationally-supported disk (of gas and star), and a bulge, with independent parameters describing each of the structural components. The distribution of the dark matter halo follows a Hernquist profile (Hernquist 1990), with a concentration parameter of $C=14$ in both galaxies following Dolag et al. (2004). We also assume a thin disk structure of gas and stars with an exponential surface density. The scale length of disk, $R_{\mathrm{d}}$, is determined following Graham $\&$ Worley (2008), and the bulge distribution is assumed to be spherical while also following a Hernquist profile. We adopt a conventionally determined disk scale height $z_{0}$, and a bulge scale length $b$ as $b=z_{0}=0.2 R_{\mathrm{d}}$. The baryon fraction derived from Komatsu et al. (2011) is used. The gas fraction of our model galaxies is consistent with the estimation in Binney \& Merrifield (1998) and Kannappan (2004). The maximum speed of the rotation curve is about $150 \mathrm{~km} \mathrm{~s}^{-1}$ as suggested by the baryonic Tully-Fisher relation (McGaugh 2005). We limit our study to Sa and $\mathrm{Sb}$ model galaxies. To consider unequal-mass mergers, we constructed three satellite galaxies by changing the total mass of the $\mathrm{Sb}$ galaxy while fixing the other parameters. They are onethird, one-sixth, and one-tenth of the Sb galaxy in total mass and are labeled as $\mathrm{Sb}_{3}, \mathrm{Sb}_{6}$, and $\mathrm{Sb}_{10}$, respectively. Table 1 summarizes the parameters of each galaxy.

The simulations were performed using the publicly-available code GADGET2 (Springel 2005) with added prescriptions for gas cooling, star formation, and feedback from Type Ia and II supernovae (SNe). We note that gas particles with $T<2 \times 10^{4} \mathrm{~K}$, number density $n>0.1 \mathrm{~cm}^{-3}$, overdensity $\Delta \rho_{\text {gas }}>100$, and $\nabla \cdot v<0$ form stars according to the standard star formation prescription: $\mathrm{d} \rho_{*} / \mathrm{d} t=c_{*} \rho_{\text {gas }} / t_{\text {dyn }}$, where $\rho_{*}$ refers to the stellar density, $t_{\text {dyn }}$ is the local dynamical timescale of gas, and $c_{*}$ is the star formation efficiency. More than a million particles were used for each run. Consequently, the particle masses are $m_{\mathrm{DM}}=4 \times 10^{5} M_{\odot}, m_{\mathrm{disk}}=m_{\mathrm{gas}}=5 \times 10^{4} M_{\odot}$, and $m_{\text {bulge }}=1.5 \times 10^{5} M_{\odot}$ for dark matter, disk, gas, and bulge, respectively. The gravitational softening lengths are $\epsilon_{\mathrm{DM}}=0.1 \mathrm{kpc}$, $\epsilon_{\text {disk }}=\epsilon_{\text {gas }}=0.2 \mathrm{kpc}$, and $\epsilon_{\text {bulge }}=0.1 \mathrm{kpc}$.

The stability of our model galaxies is measured by investigating the star formation rate (SFR) of each galaxy in isolation as shown in Fig. 2. We found SFRs of, on average, $\sim 0.22 M_{\odot} \mathrm{yr}^{-1}$ and $\sim 1.3 M_{\odot} \mathrm{yr}^{-1}$ over $1 \mathrm{Gyr}$ for the $\mathrm{Sa}$ and $\mathrm{Sb}$ models, respectively. We note that the SFRs are consistent with the observational sample of James et al. (2004).

The difference between the SFR amplitudes of the two model galaxies is due to the difference between the initial masses of gas in the disks (see Table 1). The gradual decay of SFR is as expected since the available gas is progressively converted into stars. External accretion of gas could be considered to reproduce the constant average SFRs found in observations of spiral 
Table 1. Initial parameters of model galaxies.

\begin{tabular}{lccccc}
\hline \hline & $\mathrm{Sa}$ & $\mathrm{Sb}$ & $\mathrm{Sb}_{3}$ & $\mathrm{Sb}_{6}$ & $\mathrm{Sb}_{10}$ \\
\hline Total mass, $M_{\text {vir }}\left(10^{10} M_{\odot}\right)$ & 17.0 & 17.0 & 5.7 & 2.8 & 1.7 \\
DM mass, $M_{\mathrm{DM}}\left(10^{10} M_{\odot}\right)$ & 14.1 & 14.1 & 4.7 & 2.4 & 1.4 \\
Stellar disk mass, $M_{\mathrm{d}}\left(10^{10} M_{\odot}\right)$ & 1.61 & 1.81 & 0.60 & 0.30 & 0.18 \\
Stellar bulge mass, $M_{\mathrm{b}}\left(10^{9} M_{\odot}\right)$ & 10.74 & 4.52 & 1.51 & 0.75 & 0.45 \\
Gas mass, $M_{\text {gas }}\left(10^{9} M_{\odot}\right)$ & 1.41 & 5.65 & 1.88 & 0.94 & 0.57 \\
$\mathrm{~B} / \mathrm{T}$ ratio & 0.4 & 0.2 & 0.2 & 0.2 & 0.2 \\
Spin parameter, $\lambda$ & 0.05 & 0.05 & 0.05 & 0.05 & 0.05 \\
Disk scale length, $R_{\mathrm{d}}(\mathrm{kpc})$ & 2.58 & 3.29 & 2.28 & 1.81 & 1.52 \\
\hline
\end{tabular}

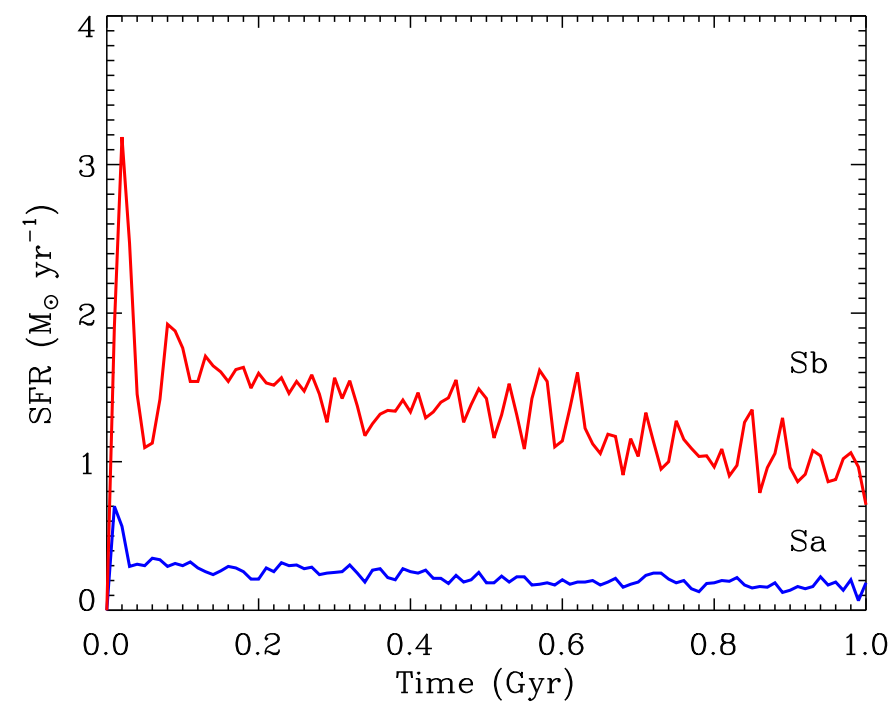

Fig. 2. Star formation rate for isolated $\mathrm{Sa}$ and $\mathrm{Sb}$ models at intervals of $10 \mathrm{Myr}$. The $\mathrm{Sb}$ model with a higher gas content shows star formation rates roughly an order of magnitude higher than the Sa model. Initial bursts of new stars in both models are contributed from the gas compression of gas into density waves and the absence of supernova feedback from young stellar populations.

galaxies in the middle of the Hubble sequence (Kennicutt 1983; Kennicutt et al. 1994), but is not included in our study in order to simplify our simulation.

As shown in Fig. 2, initial bursts of new stars occur as a result of gas compression into density waves early in the simulations. However, the initial bursts are suppressed after supernova feedbacks are exerted on gas (see Di Matteo et al. 2007). Each model galaxy is quite stable as no spurious effects are seen in the evolution of the SFRs because of specific choices of gravitational smoothing or the phase of the initial condensation of gas.

\subsection{Definition of merger timescales}

Figure 3 shows characteristic timescales of a merger event. Each merger event is characterized by several successive and distinct phases. We identify several critical timescales that show distinctive features during the merger: the first passage time $\left(t_{\mathrm{FP}}\right)$ when the companion galaxy first passes the closest distance, the maximum separation time $\left(t_{\mathrm{MS}}\right)$ after the first passage time, and the final coalescence time $\left(t_{\mathrm{FC}}\right)$ when the two galaxies finally merge. In addition, we adopt the merger-feature time $\left(t_{\mathrm{MF}}\right)$ when the merger remnant hides post-merger features viewed perpendicular to and parallel to the orbital plane. We consider two merger-feature times by visual inspection based on two surface brightness limits in the SDSS $r$ band: $\mu_{r \text {,limit }}=25\left(t_{\mathrm{MF}, 25}\right)$
Table 2. Initial orbital configurations.

\begin{tabular}{|c|c|c|c|c|}
\hline Simulation $^{a}$ & Pair $^{b}$ & $e^{c}$ & $R_{\text {peri }}{ }^{d}$ & $i_{\text {host }}^{e}$ \\
\hline SbSb0p & $\mathrm{Sb}-\mathrm{Sb}$ & 1 & 5 & 0 \\
\hline SbSb45p & $\mathrm{Sb}-\mathrm{Sb}$ & 1 & 5 & 45 \\
\hline SbSb90p & $\mathrm{Sb}-\mathrm{Sb}$ & 1 & 5 & 90 \\
\hline SbSb135p & $\mathrm{Sb}-\mathrm{Sb}$ & 1 & 5 & 135 \\
\hline SbSb180p & $\mathrm{Sb}-\mathrm{Sb}$ & 1 & 5 & 180 \\
\hline $\mathrm{SaSb} 45 \mathrm{p}$ & $\mathrm{Sa}-\mathrm{Sb}$ & 1 & 5 & 45 \\
\hline SaSb90p & $\mathrm{Sa}-\mathrm{Sb}$ & 1 & 5 & 90 \\
\hline SaSb135p & $\mathrm{Sa}-\mathrm{Sb}$ & 1 & 5 & 135 \\
\hline $\mathrm{SbSb} 45 \mathrm{r}$ & $\mathrm{Sb}-\mathrm{Sb}$ & 1 & 0 & 45 \\
\hline SbSb45p+ & $\mathrm{Sb}-\mathrm{Sb}$ & 1 & 10 & 45 \\
\hline $\mathrm{SbSb} 45 \mathrm{e}$ & $\mathrm{Sb}-\mathrm{Sb}$ & 0.95 & 5 & 45 \\
\hline $\mathrm{SbSb} 45 \mathrm{~h}$ & $\mathrm{Sb}-\mathrm{Sb}$ & 1.05 & 5 & 45 \\
\hline $\mathrm{SbSb}_{3} 45 \mathrm{~h}$ & $\mathrm{Sb}-\mathrm{Sb}_{3}$ & 1.05 & 5 & 45 \\
\hline $\mathrm{SbSb}_{6} 45 \mathrm{~h}$ & $\mathrm{Sb}-\mathrm{Sb}_{6}$ & 1.05 & 5 & 45 \\
\hline $\mathrm{SbSb}_{10} 45 \mathrm{~h}$ & $\mathrm{Sb}-\mathrm{Sb}_{10}$ & 1.05 & 5 & 45 \\
\hline SbSb45hC & $\mathrm{Sb}-\mathrm{Sb}$ & $0.95^{f}$ & $472^{f}$ & - \\
\hline
\end{tabular}

Notes. ${ }^{(a)}$ Types of galaxies, initial encounter angle, orbital shape ("p" for parabolic, "r" for radial, "e" for elliptical, "h" for hyperbolic, and "+" for larger pericentric distance), and additional cluster potential ("C" for cluster potential) are marked in the simulation name. ${ }^{(b)}$ Hostcompanion pair. ${ }^{(c)}$ Eccentricity of orbit. ${ }^{(d)}$ Pericentric distance $(\mathrm{kpc})$. ${ }^{(e)}$ Inclination of host galaxy $\left({ }^{\circ}\right) .(f)$ The orbital parameters with respect to a cluster potential.

and $28 \mathrm{mag} \mathrm{arcsec}^{-2}\left(t_{\mathrm{MF}, 28}\right)$. The $\mu_{\mathrm{r}, \text { limit }}=25 \mathrm{mag} \operatorname{arcsec}^{-2}$ is comparable to the observational limit of the SDSS. The characteristic times are listed in Table 3.

\subsection{Initial orbital configurations}

In all simulations, the initial separation between the host and the companion is $90 \mathrm{kpc}$, the virial radius of the host galaxy. The pericentric distances adopted here are consistent with those of dark matter haloes in cosmological simulations (see Khochfar \& Burkert 2006). The definition of inclination with respect to the orbital plane is adopted from Toomre \& Toomre (1972).

Table 2 summarizes all information related to the specific orbital configuration of each merger. For the convenience of comparison between the simulations, we first constructed five fiducial mergers (top five mergers in Table 2). The progenitor galaxies are $\mathrm{Sb}$ models. The eccentricity is $e=1$, and the pericentric distance is $R_{\text {peri }}=5 \mathrm{kpc}$. The inclinations of the host galaxies are set to $i_{\text {host }}=0^{\circ}$ (prograde), $45^{\circ}, 90^{\circ}, 135^{\circ}$, and $180^{\circ}$ (retrograde).

Modifying the fiducial mergers, we constructed eleven additional mergers. First, the host galaxy was altered from an $\mathrm{Sb}$ to an Sa galaxy, and the inclinations of the host galaxies are 
Table 3. Characteristic times and merger-feature time to final coalescence time ratio.

\begin{tabular}{|c|c|c|c|c|c|c|c|}
\hline Simulation & $t_{\mathrm{FP}}(\mathrm{Gyr})^{a}$ & $t_{\mathrm{MS}}(\mathrm{Gyr})^{b}$ & $t_{\mathrm{FC}}(\mathrm{Gyr})^{c}$ & $t_{\mathrm{MF}, 25}(\mathrm{Gyr})^{d}$ & $t_{\mathrm{MF}, 28}(\mathrm{Gyr})^{e}$ & $t_{\mathrm{MF}, 25} / t_{\mathrm{FC}}^{f}$ & $t_{\mathrm{MF}, 28} / t_{\mathrm{FC}}{ }^{g}$ \\
\hline \multicolumn{8}{|c|}{ Equal-mass mergers in isolated environment } \\
\hline $\mathrm{SbSb0p}$ & 0.41 & 0.71 & 1.07 & $2.43 \pm 0.34$ & $6.65 \pm 0.66$ & $2.27 \pm 0.32$ & $6.22 \pm 0.62$ \\
\hline $\mathrm{SbSb} 45 \mathrm{p}$ & 0.41 & 0.65 & 1.07 & $2.31 \pm 0.15$ & $7.52 \pm 0.74$ & $2.16 \pm 0.14$ & $7.03 \pm 0.69$ \\
\hline $\mathrm{SbSb} 90 \mathrm{p}$ & 0.41 & 0.76 & 1.30 & $3.37 \pm 0.15$ & $5.70 \pm 0.45$ & $2.59 \pm 0.11$ & $4.38 \pm 0.34$ \\
\hline SbSb135p & 0.41 & 0.60 & 1.13 & $2.44 \pm 0.12$ & $6.13 \pm 0.84$ & $2.16 \pm 0.10$ & $5.43 \pm 0.84$ \\
\hline SbSb180p & 0.41 & 0.65 & 1.03 & $2.10 \pm 0.24$ & $5.41 \pm 0.42$ & $2.04 \pm 0.24$ & $5.25 \pm 0.41$ \\
\hline SaSb45p & 0.41 & 0.67 & 1.09 & $2.40 \pm 0.33$ & $5.63 \pm 0.65$ & $2.20 \pm 0.30$ & $5.17 \pm 0.59$ \\
\hline SaSb90p & 0.41 & 0.90 & 1.41 & $2.59 \pm 0.22$ & $4.82 \pm 0.25$ & $1.83 \pm 0.16$ & $3.42 \pm 0.18$ \\
\hline SaSb135p & 0.41 & 0.63 & 1.08 & $2.26 \pm 0.20$ & $4.99 \pm 0.24$ & $2.09 \pm 0.19$ & $4.62 \pm 0.22$ \\
\hline $\mathrm{SbSb} 45 \mathrm{r}$ & 0.38 & 0.51 & 0.73 & $2.25 \pm 0.26$ & $7.01 \pm 0.26$ & $3.08 \pm 0.35$ & $9.60 \pm 0.35$ \\
\hline $\mathrm{SbSb} 45 \mathrm{p}+$ & 0.43 & 1.00 & 1.93 & $3.04 \pm 0.18$ & $6.69 \pm 0.32$ & $1.58 \pm 0.09$ & $3.47 \pm 0.17$ \\
\hline $\mathrm{SbSb} 45 \mathrm{e}$ & 0.49 & 0.61 & 0.84 & $2.68 \pm 0.32$ & $>8.50^{h}$ & $3.19 \pm 0.38$ & $>10.12$ \\
\hline $\mathrm{SbSb} 45 \mathrm{~h}$ & 0.35 & 0.80 & 1.57 & $2.99 \pm 0.20$ & $5.60 \pm 0.47$ & $1.90 \pm 0.13$ & $3.57 \pm 0.30$ \\
\hline Mean & $0.41 \pm 0.03$ & $0.71 \pm 0.14$ & $1.19 \pm 0.33$ & $2.57 \pm 0.82$ & $6.22 \pm 1.73$ & $2.16 \pm 0.80$ & $5.24 \pm 1.46$ \\
\hline \multicolumn{8}{|c|}{ Unequal-mass mergers in isolated environment } \\
\hline $\mathrm{SbSb}_{3} 45 \mathrm{~h}$ & 0.44 & 1.11 & 2.13 & $4.59 \pm 0.17$ & $7.26 \pm 0.66$ & $2.15 \pm 0.08$ & $3.41 \pm 0.31$ \\
\hline $\mathrm{SbSb}_{6} 45 \mathrm{~h}$ & 0.47 & 1.48 & 3.75 & $5.80 \pm 0.14$ & $8.30 \pm 0.39$ & $1.55 \pm 0.04$ & $2.21 \pm 0.11$ \\
\hline $\mathrm{SbSb}_{10} 45 \mathrm{~h}$ & 0.48 & 2.8 & 12.9 & - & - & - & - \\
\hline Mean $^{\mathrm{i}}$ & $0.46 \pm 0.02$ & $1.30 \pm 0.26$ & $2.94 \pm 1.15$ & $5.19 \pm 0.44$ & $7.78 \pm 0.77$ & $1.77 \pm 0.15$ & $2.65 \pm 0.26$ \\
\hline \multicolumn{8}{|c|}{ Merger in a cluster potential } \\
\hline SbSb45hC & 0.35 & 0.80 & 1.57 & $2.81 \pm 0.17$ & $4.02 \pm 0.17$ & $1.79 \pm 0.11$ & $2.56 \pm 0.04$ \\
\hline
\end{tabular}

Notes. ${ }^{(a)}$ First passage time. ${ }^{(b)}$ Maximum separation time. ${ }^{(c)}$ Final coalescence time. ${ }^{(d)}$ Merger-feature time based on $\mu_{r, \text { limit }}=25$ mag arcsec ${ }^{-2}$. ${ }^{(e)}$ Merger-feature time based on $\mu_{r \text {,limit }}=28 \mathrm{mag} \operatorname{arcsec}^{-2} .{ }^{(f)}$ Ratio between merger-feature time to final coalescence time based on $\mu_{r \text {,limit }}=$ $25 \mathrm{mag} \mathrm{arcsec}^{-2}$. ${ }^{(g)}$ Ratio between merger-feature time to final coalescence time timescales based on $\mu_{r, \text { limit }}=28$ mag arcsec $^{-2}$. ${ }^{(h)} \mathrm{Disturbed}$ structures are clearly visible until the end of simulation $(8.5 \mathrm{Gyr}) .{ }^{(i)}$ In this case, we consider only $\mathrm{SbSb}_{3} 45 \mathrm{~h}$ and $\mathrm{SbSb}_{6} 45 \mathrm{~h}$ mergers.
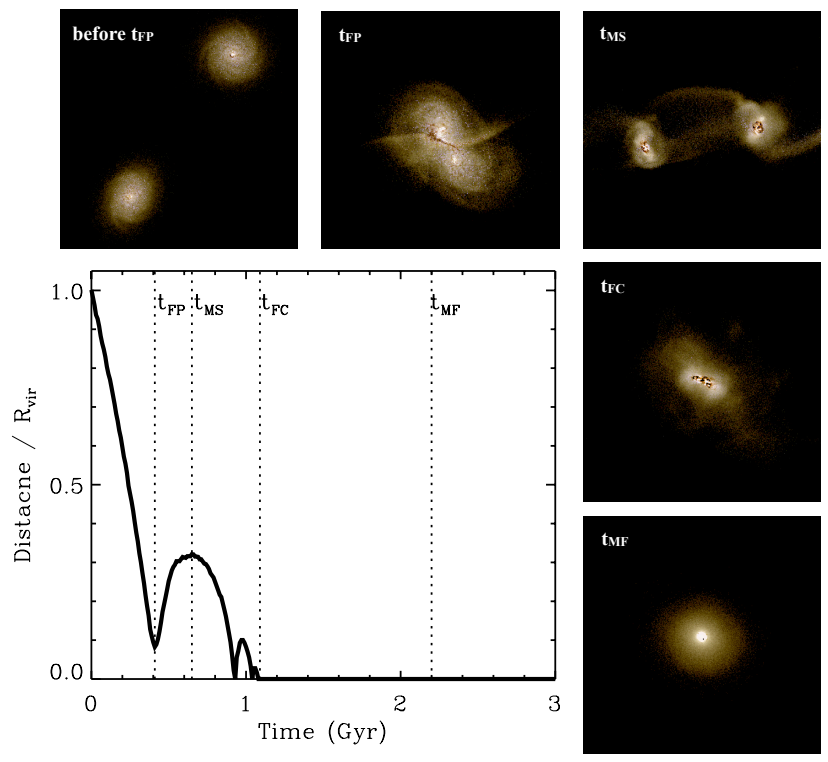

Fig. 3. Overview of merger timescales: first passage time $\left(t_{\mathrm{FP}}\right)$ when a companion or satellite galaxy first passes the closest distance, maximum separation time $\left(t_{\mathrm{MS}}\right)$ after the first passage, final coalescence time $\left(t_{\mathrm{FC}}\right)$ when two galaxies finally merge, and merger-feature time $\left(t_{\mathrm{MF}}\right)$ when post-merger features disappear given a surface brightness limit.

$i_{\text {host }}=45^{\circ}, 90^{\circ}$, and $135^{\circ}$. Second, the pericentric distance of the SbSb45p merger was changed so that $R_{\text {peri }}=0$ and $10 \mathrm{kpc}$. Third, the orbital eccentricity of the $\mathrm{SbSb} 45 \mathrm{p}$ merger was varied to consider elliptical and hyperbolic orbits as well: we chose $e=0.95$ and 1.05 . Fourth, we changed the total mass of the companion galaxy in the SbSb45h merger so that mass ratios are $3: 1$, $6: 1$, and 10:1. We chose the SbSb45h merger for comparison because its merger timescales are shorter than those in other cases and so better suited for demonstrating the effects of merger conditions in question.

As the last step, we placed a merger remnant in a rigid dark matter potential following a Hernquist profile with a Virgo-like mass of $M_{\mathrm{vir}} \simeq 4.2 \times 10^{14} M_{\odot}$, a concentration parameter of $C=9$, and a scale length of $a=236 \mathrm{kpc}$. We chose the merger remnant of the $\mathrm{SbSb} 45 \mathrm{~h}$ merger at the final coalescence time and let the merger remnant orbit in the cluster potential; the initial distance from the center is $1200 \mathrm{kpc}$ which is the virial radius of the cluster halo potential, and the pericentric distance is twice the scale length of the potential. The choice of the merger in a cluster halo environment is largely arbitrary, and we would only like to check out the environmental effect in rough senses. In all, we simulated and analyzed sixteen merger events considering different host-companion pairs, inclinations, and orbits. All equal-mass mergers are simulated until 8.5 Gyr corresponding to $\sim 8$ times the dynamical timescale at the virial radius of the host galaxy $\left(t_{\mathrm{dyn}} \sim 1.1 \mathrm{Gyr}\right)$. Since unequal-mass mergers evolve more slowly than equal-mass mergers, they are run for $13 \mathrm{Gyr}$, instead.

\subsection{Star formation}

As mentioned above, star formation in this study is intended to follow the empirical relation described in Kennicutt (1998). Thus, to investigate the validity of our models, we also test the time evolution of the surface density of the gas and the star formation surface density averaged in the central region of each galaxy.

Figure 4 shows the evolution of our model galaxies in isolation and one merger process (SbSb45p). Each symbol denotes the properties of a galaxy at an epoch given by the color key. 


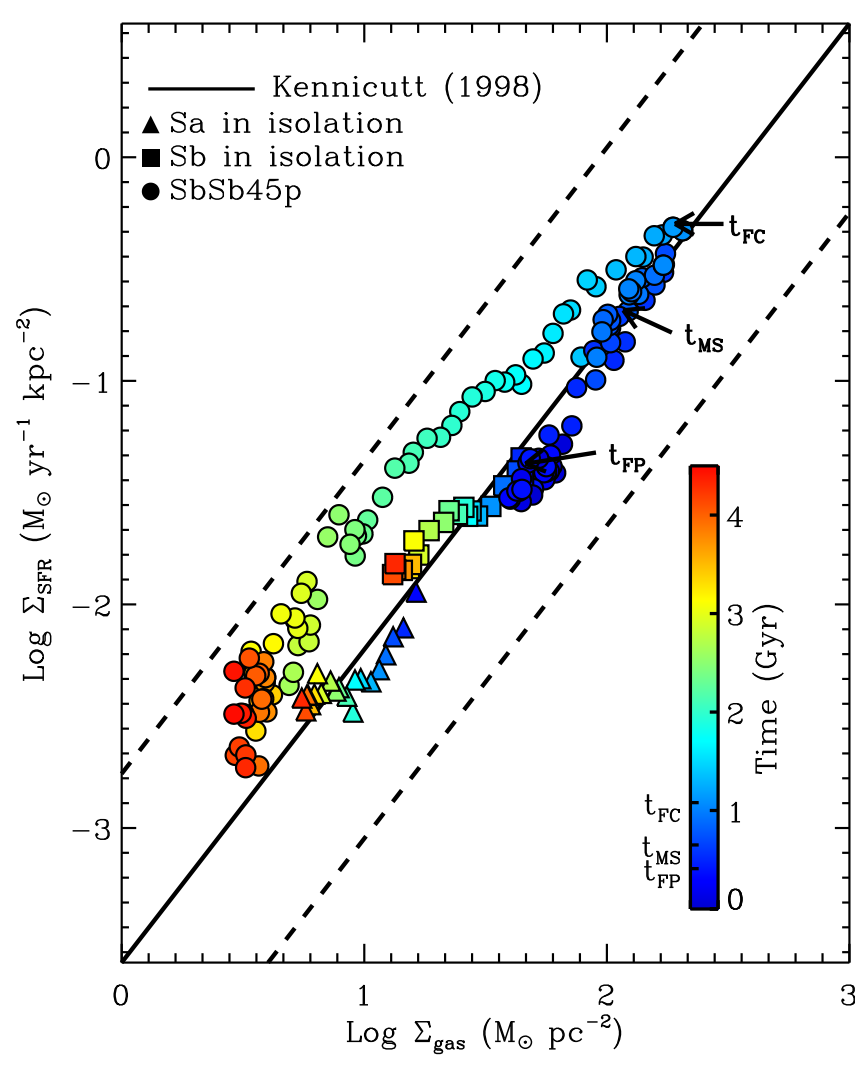

Fig. 4. Evolution of star formation surface density vs. gas column density for the Sa model (filled triangle), Sb model (filled square), and SbSb45p merger (filled circle). Each symbol corresponds to the center of a galaxy at 20 and 5 Myr time intervals for each isolated progenitor ( $\mathrm{Sa}$ and $\mathrm{Sb}$ ) and the $\mathrm{SbSb} 45 \mathrm{p}$ merger. For the $\mathrm{SbSb} 45 \mathrm{p}$ merger, the merger timescales associated with star formation are labeled with arrows: i.e., the first passage time $\left(t_{\mathrm{FP}}\right)$, the maximum separation time $\left(t_{\mathrm{MS}}\right)$, and the final coalescence time $\left(t_{\mathrm{FC}}\right)$. The solid line is the best fit given by Kennicutt (1998), and the dashed lines represent a factor of 7 upper/lower bound which covers all observations in the same literature.

In the isolated model galaxies, the surface density of gas and the star formation surface density decrease continuously starting from $\left(\log \Sigma_{\text {gas }}, \log \Sigma_{\mathrm{SFR}}\right) \sim\left(1.2 M_{\odot} \mathrm{pc}^{-2},-2 M_{\odot} \mathrm{yr}^{-1} \mathrm{pc}^{-2}\right)$ and $\left(\log \Sigma_{\text {gas }}, \log \Sigma_{\mathrm{SFR}}\right) \sim\left(1.7 M_{\odot} \mathrm{pc}^{-2},-1.3 M_{\odot} \mathrm{yr}^{-1} \mathrm{pc}^{-2}\right)$ for the $\mathrm{Sa}$ and $\mathrm{Sb}$ models, respectively. These values closely track the empirical relation in Kennicutt (1998).

The SbSb45p merger, on the other hand, shows that the surface density of gas and the star formation surface density increase as the progenitor galaxies interact and merge with each other. During the merger-induced starburst, the gas surface density and the star formation surface density reach (log $\left.\Sigma_{\text {gas }}, \log \Sigma_{\mathrm{SFR}}\right) \sim\left(2.3 M_{\odot} \mathrm{pc}^{-2},-0.2 M_{\odot} \mathrm{yr}^{-1} \mathrm{pc}^{-2}\right)$ which are comparable to the starburst galaxies with $\left(\log \Sigma_{\text {gas }}, \log \Sigma_{\mathrm{SFR}}\right) \gtrsim$ $\left(2 M_{\odot} \mathrm{pc}^{-2},-1 M_{\odot} \mathrm{yr}^{-1} \mathrm{pc}^{-2}\right)$ in Kennicutt (1998). Figure 4 also indicates that the SbSb45p merger evolves within the observational envelope. In addition, we confirm that there is good agreement between all merger simulations and the observational envelope. Therefore, we conclude that the model galaxies and the mergers discussed in this study closely follow the empirical star formation law, hence passing a sanity check regarding the sensibility of the star formation prescription in our models.

\section{Analysis}

\subsection{Modeling 2D synthetic images}

In this section, we describe our ray-tracing algorithm which allows the extraction of observable quantities from snapshots of merger simulations. This method requires information about the positions of all particles, the ages of stellar components, gas properties and chemical composition.

First, we constructed 2D regular Cartesian grids using the positional information of the particles. The spectral energy distribution (SED) is assigned to all stellar particles (both disk and bulge) following Bruzual \& Charlot (2003). Since our aim is to study the time evolution of merging galaxies at low redshift, potentially displaying resolved features, we assume a solar metallicity and 2 Gyr-old stellar components following Gallazzi et al. (2005). It is worth mentioning that dust has a critical role in diminishing the flux emitted by stars as the wavelength becomes shorter. Although the gas column density determines the strength of dust attenuation, the line-of-sight distribution of gas and stars should be considered for a more realistic approach. We use the empirical fitting formula from Bohlin et al. (1978) and the dust extinction curve provided by Calzetti et al. (2000) to calculate the amount of dust extinction from hydrogen column density. In their studies, the dust-to-gas ratios convolved with metal abundances are similar to those of the Milky Way (Alton et al. 1998; Davies et al. 1999).

To construct a mock image, we determined the field of view (FOV), distance to a galaxy, and plate scale. We assume a luminosity distance, $d_{L}=100 \mathrm{Mpc}$, which represents a typical distance to nearby galaxies in the widely used large-scale surveys such as the SDSS. In addition, we assume a plate scale of 0.5 arcsec per pixel which is comparable to the SDSS plate scale ( $\sim 0.4$ arcsec per pixel), and a FOV of 6.8 arcmin which can physically cover the merging area. In this case, 0.5 arcsec and 6.8 arcmin correspond to $0.24 \mathrm{kpc}$ and $200 \mathrm{kpc}$, respectively. In the following sections, we analyze the observational properties of galaxy mergers using the SDSS filter system.

\subsection{Convergence tests}

Before presenting our main results, it is necessary to conduct a series of convergence tests to estimate their robustness.

\subsubsection{Number of particles}

First, we check to what extent the results are affected by the mass resolution. For this purpose, we ran the SaSb45p merger three times while varying the total number of particles used to model each galaxy/halo component, decreasing the number by a factor of five and increasing by a factor two. We changed the softening lengths to be inversely proportional to the number of particles, $N$. We used $N^{-1 / 3}$ relation in this study (see Merritt 1996). The gravitational softening lengths in the lowerresolution $\mathrm{SaSb} 45 \mathrm{p}$ are $\epsilon_{\mathrm{DM}}=0.17 \mathrm{kpc}, \epsilon_{\mathrm{disk}}=\epsilon_{\mathrm{gas}}=0.34 \mathrm{kpc}$, and $\epsilon_{\text {bulge }}=0.71 \mathrm{kpc}$, while those in the higher resolution SaSb45p are $\epsilon_{\mathrm{DM}}=0.079 \mathrm{kpc}, \epsilon_{\mathrm{disk}}=\epsilon_{\mathrm{gas}}=0.16 \mathrm{kpc}$, and $\epsilon_{\text {bulge }}=0.079 \mathrm{kpc}$. Figure 5 shows the SFR and the time evolution of the amount of newly-formed stars for each simulation. We note that, despite slight differences, the SFRs all follow the same trend. Moreover, the difference between the final masses of new stars at $t=3 \mathrm{Gyr}$ is less than six percent, which suggests that our results are stable. 


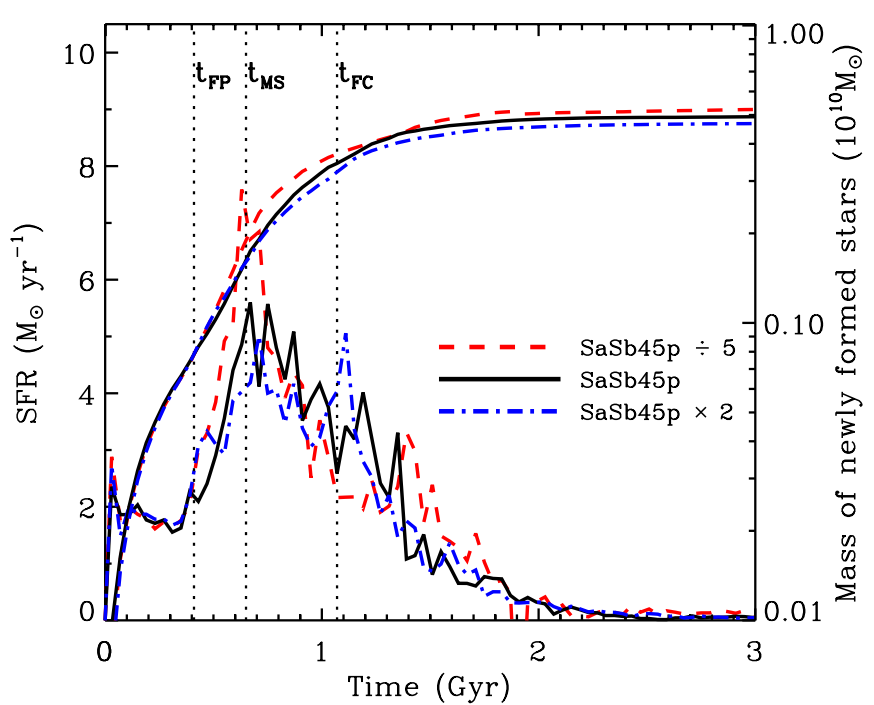

Fig. 5. Star formation rate and cumulative amount of newly formed stars for various resolutions of the SaSb45p merger: the SaSb45p merger (black solid line), five times fewer particles than the SaSb45p merger (red dashed line), and two times more particles than the SaSb45p merger (blue dash-dotted line).

\subsubsection{Plate scale}

It is also important to determine the optimal resolution at which we should perform the ray-tracing for the best accuracy in our predictions. Increased resolution requires more memory space and a longer computation time. In addition, finding a reasonable grid scale is the key to success in ray-tracing since, in this study, the physical size of the gas particle is ignored while we are able to assume stellar components as point sources.

Several studies on the internal extinction of spiral galaxies have been performed in the past decades (Giovanelli et al. 1994; Tully et al. 1998; Xilouris et al. 1999; Masters et al. 2003). The amount of dust attenuation is quantified through modeling an isolated galaxy. Methods for this computation can be put into two categories: analytical approximations (Byun et al. 1994; Baes \& Dejonghe 2001; Tuffs et al. 2004) and Monte Carlo calculations (Bianchi et al. 1996; Matthews \& Wood 2001; Pierini et al. 2004). However, models presented in these previous studies do not include galactic substructures such as spiral arms and clumps of gas which are expected to affect the total amount of dust attenuation (Corradi et al. 1996; Rocha et al. 2008). Therefore, taking into account the presence of these substructures in the ray-tracing would be a necessary step toward realism.

We estimated the amount of dust attenuation using isolated late-type galaxies where the dependences of inclinations of galaxies are clear (see, e.g., Byun et al. 1994). Figure 6 shows the amount of dust extinction in the $B$ and $J$ bands in the Sb model galaxy as a function of inclination and grid resolution. In particular, we compare the extinction slopes for six different plate scales as a function of axial ratio assuming the same luminosity distance.

First, we quantified the amount of extinction in the $B$ band with respect to the grid resolution and the ratio between the major axis $(a)$ and the minor axis $(b)$ of the Sb model galaxy. We found that, apart from the grid with the lowest resolution ( 5 arcsec per pixel), the other grid resolutions ( 0.3 to 3 arcsec per pixel) led to similar variations in dust attenuation in the $B$ bands, $\Delta M_{\mathrm{B}}$ with respect to the axial ratio $\log (a / b)$. When compared to

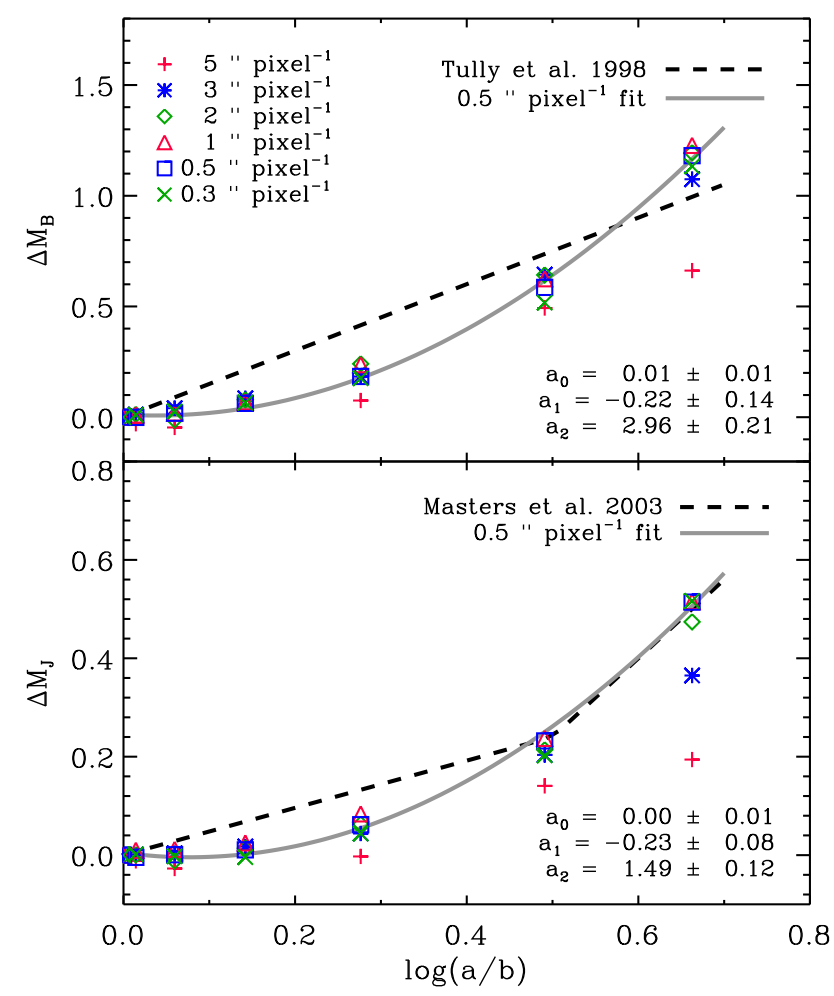

Fig. 6. Amount of dust attenuation in $B$ bands (top) and $J$ bands (bottom) with respect to the ratio between the major axis $(a)$ and the minor axis $(b)$ of the $\mathrm{Sb}$ model galaxy. Each symbol represents the attenuated magnitude in each model galaxy with a specific plate grid resolution. The dashed line in the top panel is the fitting function obtained from Tully et al. (1998), and the dashed line in the bottom panel is the bilinear fit obtained from Masters et al. (2003).

the results from Tully et al. (1998), who measured $B$ band extinctions of late-type galaxies with various inclinations, we found that our choice of a grid resolution of 0.5 arcsec per pixel agreed well with observations. Similar trends were found in the $J$ band. Masters et al. (2003) argued that dust attenuation is not a linear function of the axial ratio, but is bilinear or has a quadratic form. Masters et al. (2003) also suggest a bilinear equation of dust attenuation as a function of inclination compared to the simple linear formulation in Tully et al. (1998).

Figure 6 shows that high grid resolutions lead to a nice agreement with the observations. Low grid resolutions poorly resolve the structures which result in deviation from the fitting formula suggested by the observations. In the following, we then computed fitting functions by adopting grids of 0.5 arcsec per pixel. We fit the $B$ and $J$ band extinction with a quadratic curve defined as

$\Delta M_{\mathrm{X}}=a_{0}+a_{1} \log (a / b)+a_{2}[\log (a / b)]^{2}$,

where $a_{0}=0.01 \pm 0.01, a_{1}=-0.22 \pm 0.14$, and $a_{2}=2.96 \pm 0.21$ for the $B$ band; $a_{0}=0.00 \pm 0.01, a_{1}=-0.23 \pm 0.08, a_{2}=$ $1.49 \pm 0.12$ for the $J$ band; and $a / b$ is the axial ratio between the semimajor axis and the semiminor axis of a galaxy.

\section{Results}

\subsection{Detectability of tidal features}

For collisionless particles, the dynamical timescale in the central region of a merger remnant is relatively short because of 
I. Ji et al.: Lifetime of merger features of equal-mass disk mergers

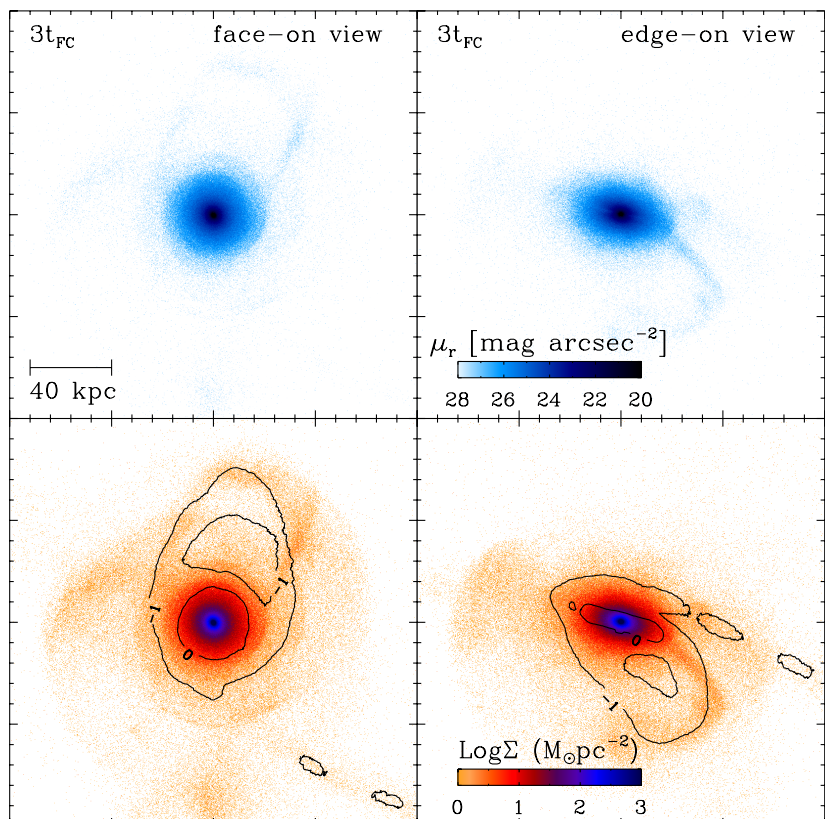

Fig. 7. Mock image and surface column density of the SbSb45h merger at three times the final coalescence time $\left(t_{\mathrm{FC}}=1.57 \mathrm{Gyr}\right)$. Top panels show mock images viewed perpendicular to (face-on) and parallel to (edge-on) the orbital plane of a companion galaxy in left-hand column and in right-hand column, respectively. Color scheme is surface brightness in the SDSS $r$ band assuming luminosity distance, $d_{L}=100 \mathrm{Mpc}$ and observational limit, $\mu_{\text {limit }}=28 \mathrm{mag} \operatorname{arcsec}^{-2}$. Bottom panels show surface column density of stellar and gaseous mass viewed perpendicular to (face-on) and parallel to (edge-on) the orbital plane of a companion galaxy in left-hand column and in right-hand column, respectively. Color scheme represents column density of stellar mass. Contour is gaseous surface density, and its values are labeled in logarithmic scale.

the violent mixture of the particles (Mihos \& Hernquist 1996; Springel 2000; Cox et al. 2006b). However, the outer region has a larger dynamical timescale which leads to long-lasting postmerger features such as shells, loops, and long tidal arms. For gas particles, they undergo shocks or dissipation of energy and angular momentum during the interaction. The gravitational torques resulting from perturbed structures induce gas to flow inwards (see also Negroponte \& White 1983). The gas inflows end up with the formation of rotating rings or blobs.

Figure 7 shows the mock images in the SDSS $r$ band (top panels) and stellar and gaseous column density (bottom panels) of the $\mathrm{SbSb} 45 \mathrm{~h}$ merger at three times the final coalescence time $\left(t_{\mathrm{FC}}=1.57 \mathrm{Gyr}\right)$. Although a few giga years elapsed after the final coalescence, post-merger features are clearly visible in the column density. Substructures of merging galaxies are stretched out along with the orbital plane forming characteristic post-merger features such as shells and loops. Although the position and shape of the features are dependent on a viewing angle, they are clearly identifiable.

However, some of the post-merger features are invisible in mock images (see Fig. 7, top panels). This is mainly because the features have low surface column density. In our simulations, stellar surface density lower than $\sim 2 M_{\odot} \mathrm{pc}^{-2}$ exceeds the surface brightness limit of $\mu_{\text {limit }}=28 \mathrm{mag} \mathrm{arcsec}^{-2}$.

\subsection{Merger-feature time of equal-mass mergers}

Figure 8 shows the mock images of the SbSb45h merger at different characteristic times and according to the observational limits of the SDSS $r$ band. The images using the SDSS $\mu_{\text {limit }}$ reveal only the central part of each galaxy, resulting in the merger remnant reaching a spheroidal shape in a relatively short period of time. For example, the faint features of the $\mathrm{SbSb} 45 \mathrm{~h}$ merger already become invisible at twice the final coalescence time $\left(t_{\mathrm{FC}} \sim 1.57 \mathrm{Gyr}\right)$ under the SDSS conditions. However, the mock images with a deeper $\mu_{\text {limit }}$ indicate that the merger remnant exhibits extended faint structures for more than two times the final coalescence time. This confirms the fact that measuring the merger-feature time of merger remnants strongly depends on $\mu_{\text {limit }}$.

To compare two merger-feature times, we have estimated the ratio of the merger-feature time to the final coalescence time, $t_{\mathrm{MF}} / t_{\mathrm{FC}}$ (see Table 3 ). We note that in contrast to the mergerfeature time based on $\mu_{\text {limit }}=25 \mathrm{mag} \operatorname{arcsec}^{-2}, t_{\mathrm{MF}, 25}$, not all merger-feature times based on $\mu_{\text {limit }}=28 \mathrm{mag} \operatorname{arcsec}^{-2}, t_{\mathrm{MF}, 28}$ could be estimated since faint features survive through the end of the simulation. Table 3 shows that $t_{\mathrm{MF}, 25} / t_{\mathrm{FC}}$ is always lower than 3 . The faint features in all merger simulations disappear by $2.16 \pm 0.80$ times the final coalescence time, or $1.38 \pm 0.88 \mathrm{Gyr}$ after the final coalescence, which is comparable to the finding of Lotz et al. (2008). However, $t_{\mathrm{MF}, 28} / t_{\mathrm{FC}}$ is approximately two times greater than $t_{\mathrm{MF}, 25} / t_{\mathrm{FC}}$ and is greater than 3 for all mergers.

We also study how merger scenarios affect $t_{\mathrm{MF}} / t_{\mathrm{FC}}$. The relaxation timescale of a merger remnant depends not only on orbital configurations (Hernandez \& Lee 2004) but also on the density of the merger remnant (Conselice 2006). Therefore, the relaxation timescales of the inner and outer regions of merger remnants are different, and the timescales are related to $t_{\mathrm{MF}, 25} / t_{\mathrm{FC}}$ or $t_{\mathrm{MF}, 28} / t_{\mathrm{FC}}$. First, the prograde-prograde merger takes longer to have its substructures relaxed and thus to hide post-merger features than the retrograde-prograde merger does. For example, one can see in Table 3 that the SbSb0p merger shows a larger value of $t_{\mathrm{MF}, 28} / t_{\mathrm{FC}}$ than the SbSb180p merger does, that is, $6.22 \pm 0.62$ vs. $5.25 \pm 0.41$, respectively ${ }^{1}$. Second, mergers having a smaller orbital angular momentum (SbSb45r and $\mathrm{SbSb} 45 \mathrm{e})$ tend to have shorter merging times $\left(t_{\mathrm{FC}}, \mathrm{Col} .4\right.$ in Table 3$)$ and longer merger-feature times ( $t_{\mathrm{MF}}$, Col. 6 in Table 3$)$ and so result in larger values of $t_{\mathrm{MF}} / t_{\mathrm{FC}}(\mathrm{Col} .8$ in Table 3$)$ than the larger-orbital-angular-momentum mergers $(\mathrm{SbSb} 45 \mathrm{p}+$ and $\mathrm{SbSb} 45 \mathrm{~h}$ ). We can perhaps interpret this as follows. A merger with low orbital angular momentum (for example, a direct headon collision) causes a rapid relaxation in the central region of the merger remnant. This makes the central region act like a point source. The outer region of the merger remnant feels looser and displays merger features for a longer period of time as a result. The essence of this interpretation is to realize that the merger timescale is determined by the structure of the central parts of the merger remnant, whereas the merger feature timescale for deep imaging conditions is derived from the outskirts. Lastly, the bulge-to-total mass ratio $(\mathrm{B} / \mathrm{T})$ affects the relaxation timescales of at least the inner regions of the merger remnants. For this reason, all values of $t_{\mathrm{MF}, 25} / t_{\mathrm{FC}}$ for the SaSb mergers are smaller than for the SbSb mergers.

1 Much of our interpretation is based on the $\mu_{\text {limit }}=28 \mathrm{mag} \operatorname{arcsec}^{-2}$ cases, because $\mu_{\text {limit }}=25 \mathrm{mag} \operatorname{arcsec}^{-2}$ cases usually reveal only the central regions of mergers which are insufficient to illustrate the whole merger effects. 


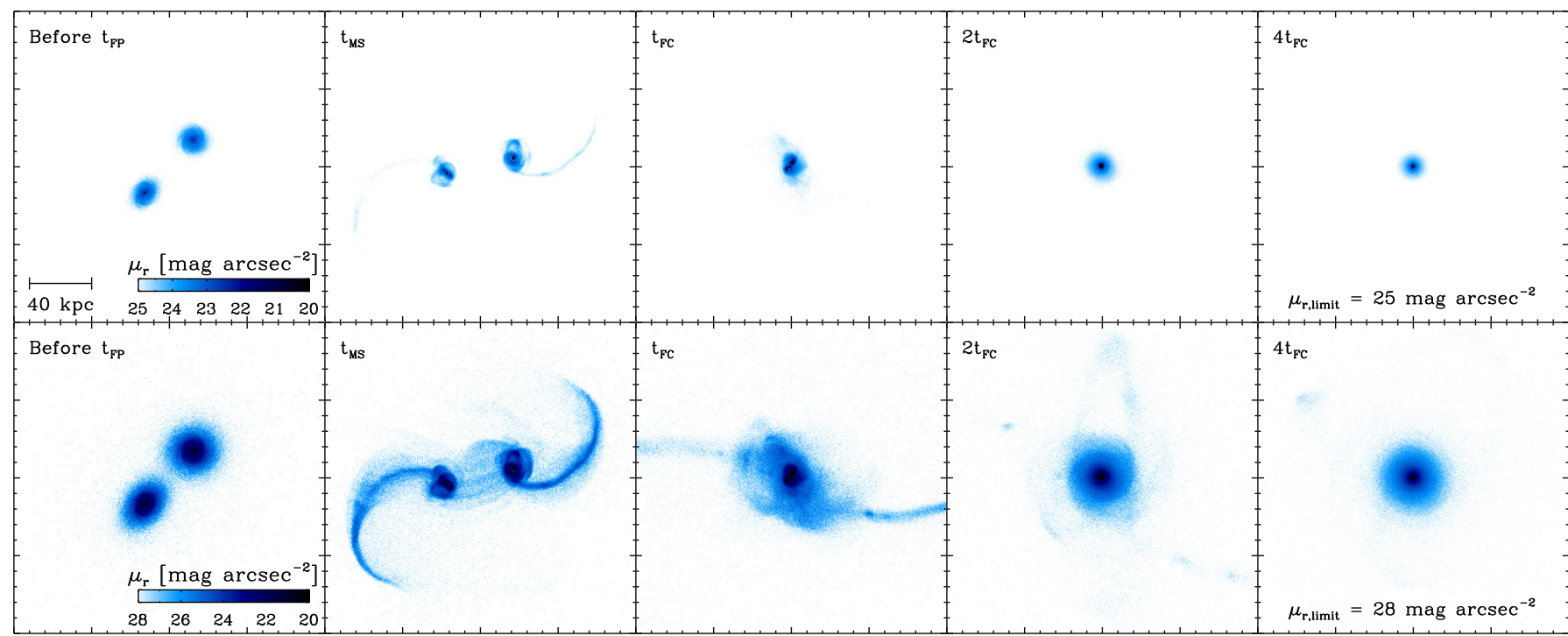

Fig. 8. Mock images of the SbSb45h merger (mass ratio is 1:1) viewed perpendicular to the orbital plane of a companion galaxy. Color scheme is surface brightness in the SDSS $r$ band assuming luminosity distance, $d_{L}=100 \mathrm{Mpc}$. Each row shows a merger process in time sequence: before the first passage time $\left(t_{\mathrm{FP}}\right)$, the maximum separation time $\left(t_{\mathrm{MS}}\right)$, and units of the final coalescence time $\left(t_{\mathrm{FC}}\right)$, from left to right. Each column is plotted with different observation limits: $\mu_{\text {limit }}=25 \mathrm{mag} \operatorname{arcsec}^{-2}$ (top) and $\mu_{\text {limit }}=28 \mathrm{mag} \mathrm{arcsec}^{-2}$ (bottom). Background color represents observation limit.

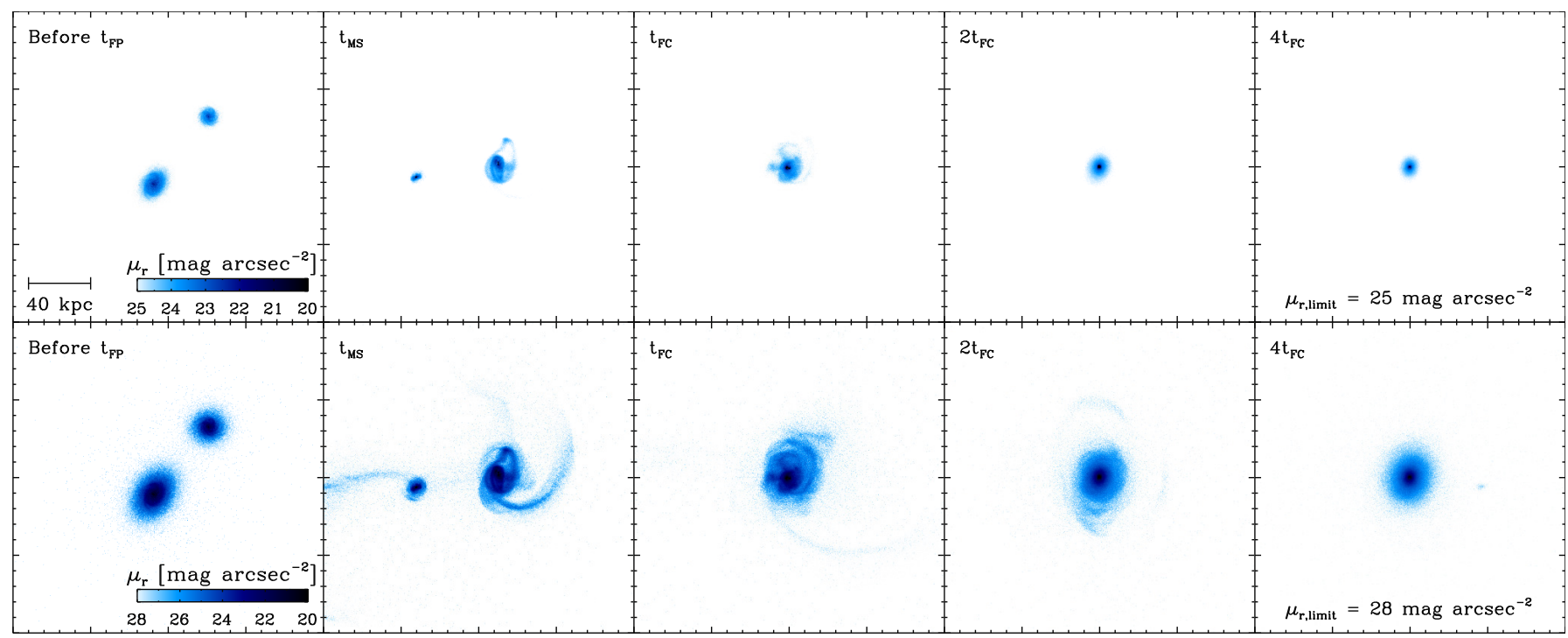

Fig. 9. Same as Fig. 8, but for the $\mathrm{SbSb}_{3} 45 \mathrm{~h}$ merger (mass ratio is 3:1).

\subsection{Effect of mass ratio}

We ran three additional mergers to qualitatively discuss the effect of mass ratio on the merger-feature time. Figures 9 and 10 show the mock images of the $\mathrm{SbSb}_{3} 45 \mathrm{~h}$ and the $\mathrm{SbSb}_{6} 45 \mathrm{~h}$ mergers, respectively. Their evolution is shown at different characteristic times and according to the observational limits of the SDSS $r$ band. For equal-mass mergers, two long and symmetric tidal arms are well developed as shown in Fig. 8. Unequal-mass mergers, however, show asymmetric tidal structures (see also Barnes 1992; Springel et al. 2005b; Lotz et al. 2008). As the mass ratio between two galaxies becomes larger (see Fig. 10), tidal bridges are unseen and tidal arms are tightly wound.

We investigate the effect of mass ratio on the merger feature time. The ratio of the merger-feature time to the final coalescence time has no clear difference from major mergers (defined as mass ratios are less than $3: 1$ ). For the $\mathrm{SbSb}_{3} 45 \mathrm{~h}$ merger, both
$t_{\mathrm{MF}, 25} / t_{\mathrm{FC}}$ and $t_{\mathrm{MF}, 28} / t_{\mathrm{FC}}$ are comparable to those of the $\mathrm{SbSb} 45 \mathrm{~h}$ merger (see Table 3 ). On the other hand, although the mergerfeature times of the $\mathrm{SbSb}_{6} 45 \mathrm{~h}$ merger (i.e., $t_{\mathrm{MF}, 25}$ and $t_{\mathrm{MF}, 28}$ ) are greater than those of the SbSb45h merger, both $t_{\mathrm{MF}, 25} / t_{\mathrm{FC}}$ and $t_{\mathrm{MF}, 28} / t_{\mathrm{FC}}$ of the $\mathrm{SbSb}_{6} 45 \mathrm{~h}$ merger are less than those of the SbSb45h merger. In essence, unequal-mass mergers show merger features for a greater amount of time mainly because they take longer to merge, compared to equal-mass mergers.

Figure 11 shows a reference case of a 10:1 merger. It is interesting to note that this "minor" merger case does not exhibit merger features clearly even before $t_{\mathrm{FC}}$. We are tempted to interpret this in order to suggest that such minor mergers, while they may be much more abundant than major mergers, may be very difficult to detect even in deep imaging conditions. Conversely, most of the merge features in today's typical surveys may very well be results of relatively "major" mergers. Confirmation of this conjecture requires a much more thorough modeling effort. 
I. Ji et al.: Lifetime of merger features of equal-mass disk mergers

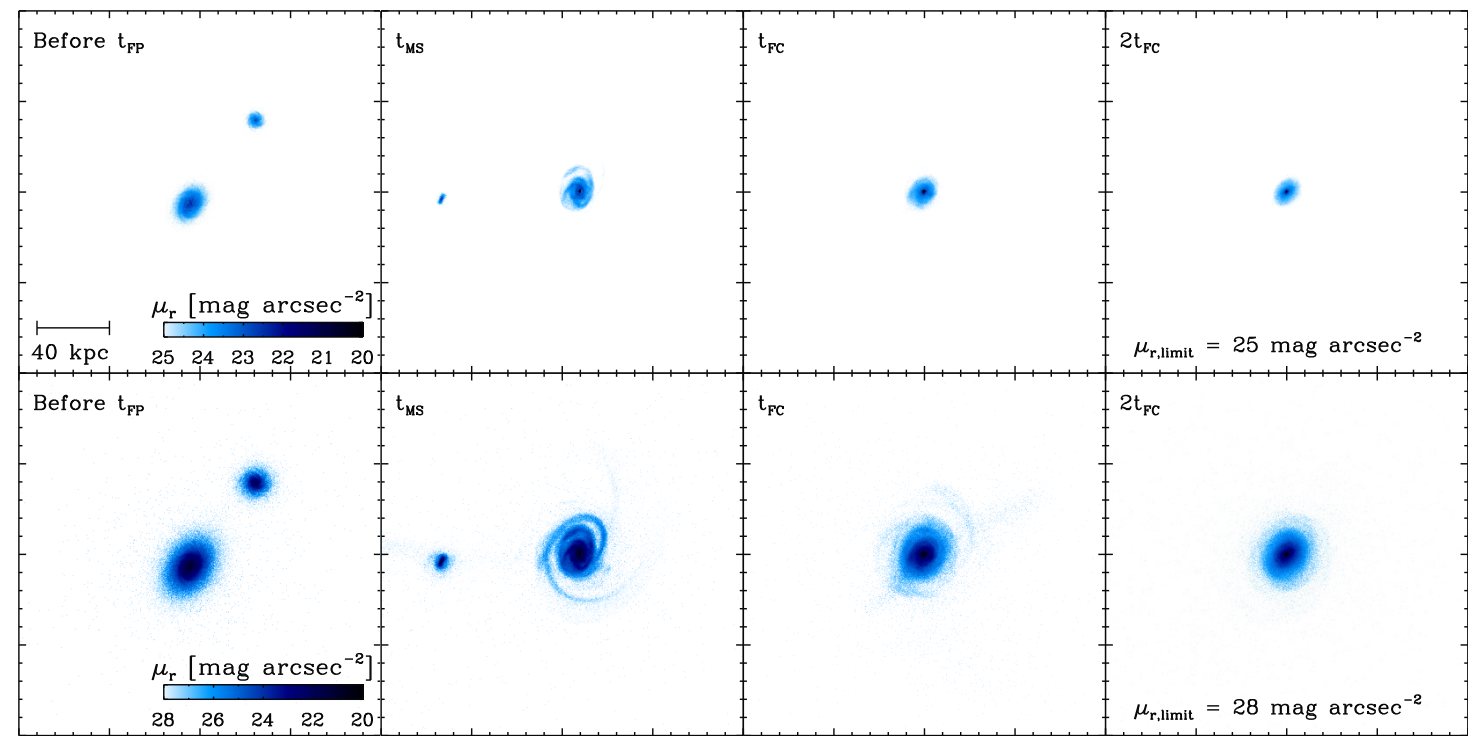

Fig. 10. Same as Fig. 8, but for the $\mathrm{SbSb}_{6} 45 \mathrm{~h}$ merger (mass ratio is $6: 1$ ).

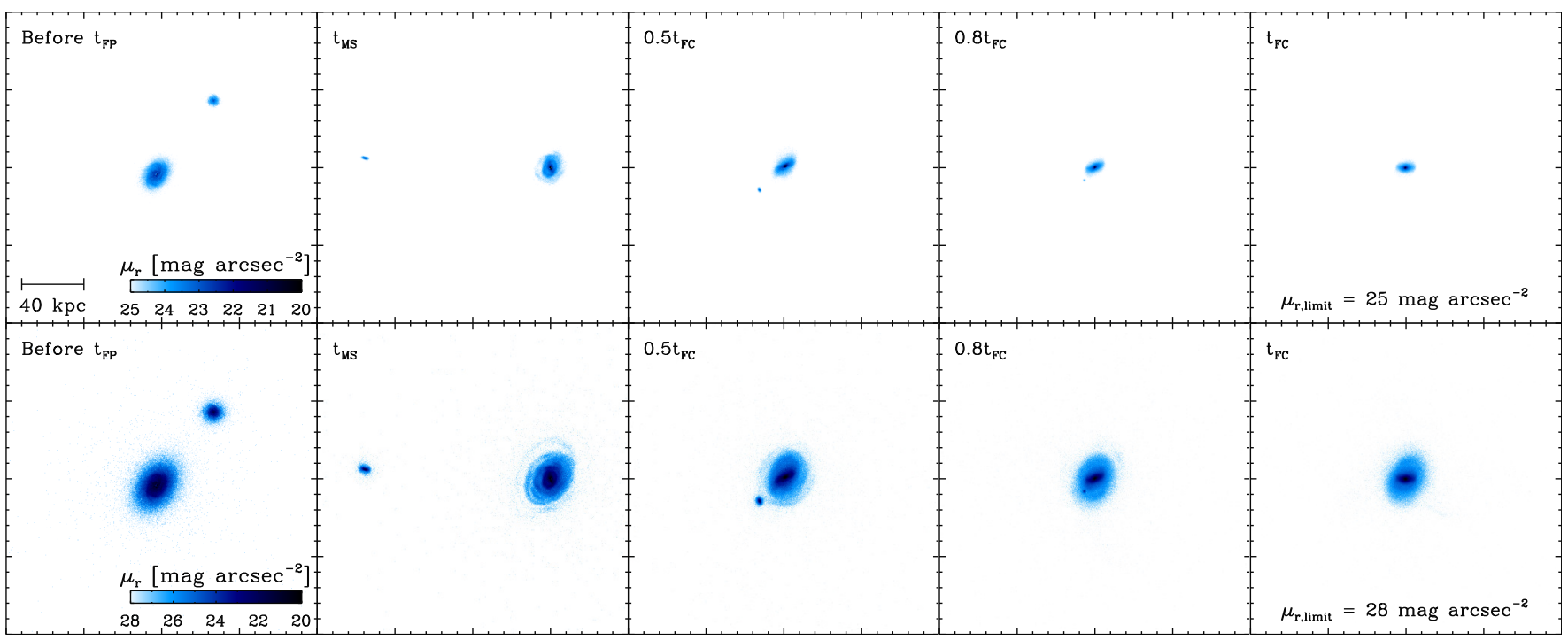

Fig. 11. Same as Fig. 8, but for the $\mathrm{SbSb}_{10} 45 \mathrm{~h}$ merger (mass ratio is 10:1).

It is also hinted in Figs. 8-11 that long symmetric tidal tails and large loops are found more frequently in major mergers (see, e.g., Wang et al. 2012). It also seems that the degree of symmetry scales to the mass ratio between the merging galaxies. It again requires many more runs filling a possible parameter space to confirm the statement, but if it is confirmed it would imply that such features can be used to find and characterize major merger remnants.

\subsection{Effect of tidal force}

We also qualitatively examined the effect of tidal force on the merger-feature time. Figure 12 shows the stellar and gaseous column density of the SbSb45hC merger at three times the final coalescence time $\left(t_{\mathrm{FC}}=1.57 \mathrm{Gyr}\right)$. Stars and gas in low-density regions of the $\mathrm{SbSb} 45 \mathrm{hC}$ merger are stripped off having undergone tidal force of a cluster potential. As a result, the stars and gas are concentrated in the central region which does not happen in an isolated environment (see Fig. 7).

Figure 13 shows the mock images of the SbSb45hC merger at twice and three times the final coalescence time and according to the observational limits of the SDSS $r$ band. We found that the merger-feature time of $\mu_{\text {limit }}=25 \mathrm{mag} \operatorname{arcsec}^{-2}$ for the SbSb45hC merger $\left(t_{\mathrm{MF}, 25}=2.81 \pm 0.17\right)$ is comparable to the SbSb45h merger $\left(t_{\mathrm{MF}, 25}=2.99 \pm 0.20\right)$. Shallow mock images of $\mu_{\text {limit }}=25 \mathrm{mag} \operatorname{arcsec}^{-2}$ mainly reveal the central region of each merger remnant which is less affected by tidal force because of its high density.

On the other hand, the effect of tidal force on post-merger features are better examined in deeper images. The mergerfeature time of the $\mathrm{SbSb} 45 \mathrm{hC}$ merger based on $\mu_{r \text {,limit }}=$ $28 \mathrm{mag} \mathrm{arcsec}^{-2}\left(t_{\mathrm{MF}, 28}=2.56 \pm 0.04\right)$ is $\sim 30 \%$ smaller than the SbSb45h merger $\left(t_{\mathrm{MF}, 28}=3.57 \pm 0.30\right)$.

\subsection{An empirical test on the SDSS database}

To test the depth dependence of merger feature lifetime, we performed a simplistic test using the SDSS database. We used the images of galaxies that are present both in the standard SDSS DR7 database and in the SDSS Stripe82 database (Abazajian et al. 2009). The surface brightness limit of Stripe82 


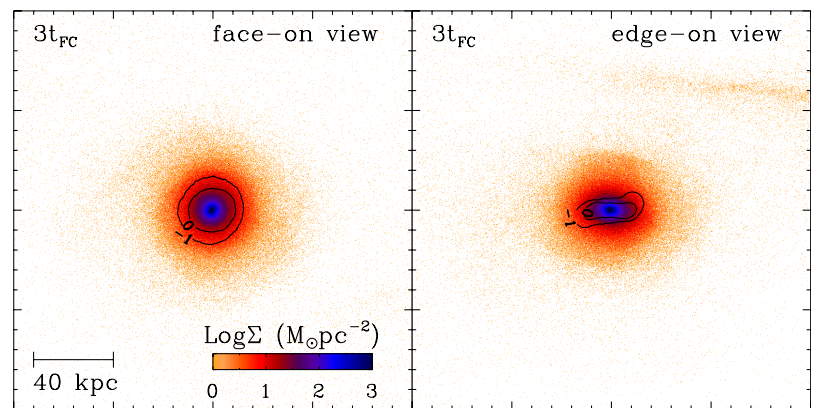

Fig. 12. Surface column density of the SbSb45hC merger at three times the final coalescence time $\left(t_{\mathrm{FC}}=1.57 \mathrm{Gyr}\right)$ viewed perpendicular to (face-on) and parallel to (edge-on) the orbital plane of a companion galaxy in left-hand column and in right-hand column, respectively. Color scheme represents column density of stellar mass. Contour is gaseous surface density, and its values are labeled in logarithmic scale.

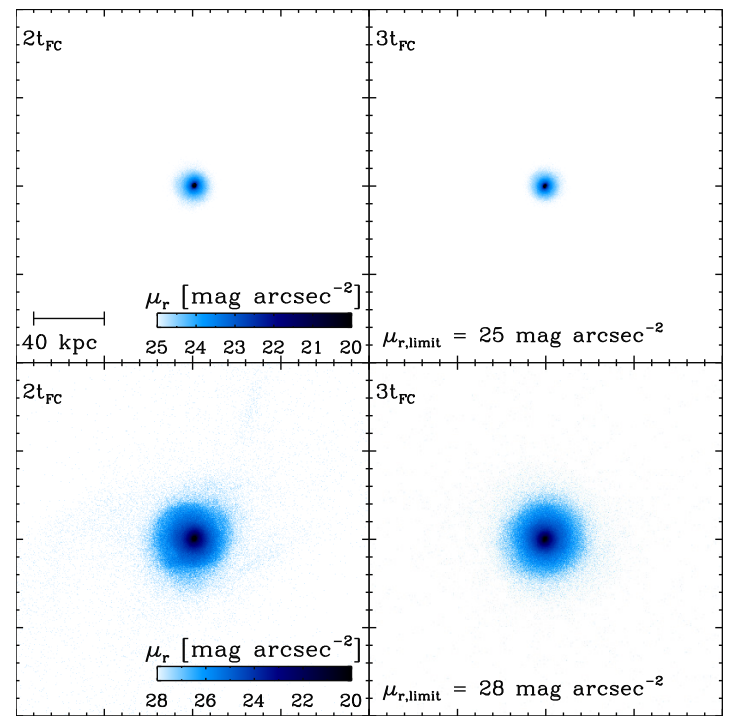

Fig. 13. Same as Fig. 8, but for SbSb45hC merger. We note that the evolution of the $\mathrm{SbSb} 45 \mathrm{hC}$ merger before the final coalescence time is as the same as the $\mathrm{SbSb} 45 \mathrm{~h}$ merger.

(27 mag arcsec -2 in $r^{\prime}$ band) is roughly 2 mag deeper than that of DR7 instead of 3 mag which was the difference we made calculations for, and mergers that can be found in SDSS galaxies are not necessarily equal-mass mergers. Thus, this comparison is not exactly suited to test our prediction, but it is the easiest test and at the least can serve as a useful milestone. We performed visual inspection on a volume-limited sample of 1453 galaxies. Among early-type galaxies, $59 \pm 4$ and $160 \pm 14$ galaxies were classified as post-merger galaxies in DR7 and Stripe82, respectively, where the errors are from multiple inspections. While it is trivial that merger features are more frequent in deeper images, we are encouraged by the fact that, as was suggested by the simulations, a larger number of galaxies by approximately a factor of three were found in Stripe82.

\section{Discussion}

In this paper, we investigate the merger-feature time of equalmass disk mergers using $N$-body/hydrodynamic simulations. To cover a realistic range of merger scenarios, we consider different orbit types, host galaxy inclinations, and the morphological properties of the two main galaxies. We ran additional merger simulations considering different mass ratios and putting the mergers in a cluster halo environment to help understand the features of equal-mass mergers.

The post-merger signatures of nearly all samples in isolation survived for more than three times the final coalescence time when we see galaxies with a deep surface brightness limit of $28 \mathrm{mag} \operatorname{arcsec}^{-2}$. The lifetime of merger features is shorter by $30 \%$ when mergers happen in a large cluster environment. So, for random environments, the merger feature lifetime can be said to be $\sim 3.5$ times the final coalescence time. This has an obvious impact on the galaxy morphology classification, especially on post-merger remnants. Sheen et al. (2012) recently found from their deep imaging $\left(\mu_{\text {limit }} \sim 28 \mathrm{mag} \mathrm{arcsec}^{-2}\right)$ campaign that a large fraction $(\sim 40 \%)$ of bulge-dominant galaxies in massive clusters show major post-merger features. Using a semianalytic model of Lee \& Yi (2013), Yi et al. (2013) interpreted it as a result of merger relics from the merging events from the past halo environments by adopting the merger-feature time estimates from the preliminary result of our study.

Even though we found and discussed noticeable differences in merger-feature time between a variety of proposed merger scenarios, our sample is still limited. For example, although equal-mass mergers are influential phenomena that clearly exhibit transitions of galaxy property, they are rare cases in the universe. Minor mergers are expected to dominate galaxy evolution over major mergers because they occur more frequently (Kaviraj et al. 2009). However, our reference models suggest hints that tidal features induced by relatively minor mergers (e.g., $m_{2} / m_{1} \lesssim 1 / 6$ ) might be difficult to detect in deep images.

Moreover, mergers of gas-rich galaxies are expected to be frequent at high redshifts. Without considering gas-rich mergers, we cannot see the full picture of galaxy evolution. Furthermore, gas-rich mergers should accompany one of the most important effects on galaxy growth, namely AGN feedback. Recent studies have shown that super massive black holes (SMBHs) in the centers of galaxies are connected to the growth of galaxies. The velocity dispersion of the bulge component is proportional to the BH mass of the galaxy (Tremaine et al. 2002). This coevolution of galaxies and SMBHs has been suggested by recent studies; for example, the correlation between star formation and $\mathrm{BH}$ accretion (Zheng et al. 2009), quasar activity of merging galaxies (Sanders et al. 1988; Hopkins et al. 2008; Li et al. 2007; Sijacki et al. 2009), and the quenching of star formation due to AGN feedback (Springel et al. 2005b; Di Matteo et al. 2005; Schawinski et al. 2006, 2007b,a; Sijacki et al. 2007; Dubois et al. 2010, 2013; Teyssier et al. 2011). For a more realistic and comprehensive study of merging galaxies we will include the effects of AGNs in our future studies. However, we think that the inclusion of AGN feedback would not affect our analysis on post-merger features much because most of the star formation induced by mergers is concentrated in the central regions of the merger remnant while post-merger features are mostly made up of older pre-existing stars in our simulations (see also Peirani et al. 2010).

We performed most of the numerical simulations assuming an isolated environment. Galaxy interactions in reality occur in various environments including galaxy clusters. In addition to galaxy mergers, there are physical mechanisms that affect the evolution of galaxies in clusters: ram-pressure stripping (Gunn \& Gott 1972), high-speed galaxy encounters (galaxy "harassment", Moore et al. 1996), tidal stripping by a cluster halo potential (Byrd \& Valtonen 1990; Valluri 1993), and thermal evaporation of cold gas inside galaxies (Cowie \& Songaila 1977). Among these mechanisms, tidal stripping plays a particularly important role in shaping merger remnants. Although 
we confirmed that the tidal structures of merger remnants are stripped off rapidly in a cluster potential (e.g., Mihos 2004), we have not checked the effect of orbital parameters of merger remnants falling into galaxy clusters yet. Therefore, the quantification of $t_{\mathrm{PM}} / t_{\mathrm{FC}}$ in various cluster environments should be investigated in future studies.

Ram-pressure stripping, and other stripping processes, affect the gas content in galaxies in deep potential wells which will in turn affect any gas-dependent properties of galaxies, star formation, for example. However, it would not significantly affect our main predictions of merger-feature times. This is because, as our simulations suggest, most of the faint merger features that are easily detected are composed of pre-existing old stars (which behave following dissipationless processes and angular momentum conservation) rather than newly formed stars (see, e.g., Fig. 4 of Peirani et al. 2010). Moreover, we do not expect that ram-pressure stripping has a significant impact on removing gas in our model galaxies $\left(M \sim 1.7 \times 10^{11} M_{\odot}\right.$ for equal-mass mergers) because it is thought to be more efficient for lower mass objects, i.e., $M<6 \times 10^{10} M_{\odot}$ (Nickerson et al. 2011). We admit that more robust calculations must ultimately include all important physical processes, such as ram-pressure stripping. But for the focus of this paper at the moment, current calculations should be sufficiently elaborate.

Acknowledgements. We thank Volker Spingel for making the Gadget code available to us. S.K.Y. acknowledges support from National Research Foundation of Korea (FY2014). Numerical simulation was performed using the KISTI supercomputer (KSC-2012-C2-11) and the KASI supercomputer. Much of this manuscript was written during the visit of SKY to University of Nottingham and University of Oxford under the travel support by the LG Yon-Am Foundation. We would like to thank the referee for his/her comments that have improved the quality of the original manuscript.

\section{References}

Abazajian, K. N., Adelman-McCarthy, J. K., Agüeros, M. A., et al. 2009, ApJS, 182,543

Alton, P. B., Trewhella, M., Davies, J. I., et al. 1998, A\&A, 335, 807

Baes, M., \& Dejonghe, H. 2001, MNRAS, 326, 733

Barnes, J. E. 1992, ApJ, 393, 484

Barnes, J. E., \& Hernquist, L. E. 1991, ApJ, 370, L65

Barton, E. J., Geller, M. J., \& Kenyon, S. J. 2000, ApJ, 530, 660

Bell, E. F., Phleps, S., Somerville, R. S., et al. 2006, ApJ, 652, 270

Bianchi, S., Ferrara, A., \& Giovanardi, C. 1996, ApJ, 465, 127

Binney, J., \& Merrifield, M. 1998, Galactic Astronomy (Princeton: Princeton University Press)

Bohlin, R. C., Savage, B. D., \& Drake, J. F. 1978, ApJ, 224, 132

Boylan-Kolchin, M., Ma, C.-P., \& Quataert, E. 2008, MNRAS, 383, 93

Bruzual, G., \& Charlot, S. 2003, MNRAS, 344, 1000

Byrd, G., \& Valtonen, M. 1990, ApJ, 350, 89

Byun, Y. I., Freeman, K. C., \& Kylafis, N. D. 1994, ApJ, 432, 114

Calzetti, D., Armus, L., Bohlin, R. C., et al. 2000, ApJ, 533, 682

Conselice, C. J. 2006, ApJ, 638, 686

Corradi, R. L. M., Beckman, J. E., \& Simonneau, E. 1996, MNRAS, 282, 1005

Cowie, L. L., \& Songaila, A. 1977, Nature, 266, 501

Cox, T. J., Dutta, S. N., Di Matteo, T., et al. 2006a, ApJ, 650, 791

Cox, T. J., Jonsson, P., Primack, J. R., \& Somerville, R. S. 2006b, MNRAS, 373 , 1013

Davies, J. I., Alton, P., Trewhella, M., Evans, R., \& Bianchi, S. 1999, MNRAS, 304, 495

De Propris, R., Liske, J., Driver, S. P., Allen, P. D., \& Cross, N. J. G. 2005, AJ, 130,1516

Di Matteo, P., Combes, F., Melchior, A.-L., \& Semelin, B. 2007, A\&A, 468, 61

Di Matteo, T., Springel, V., \& Hernquist, L. 2005, Nature, 433, 604

Dolag, K., Bartelmann, M., Perrotta, F., et al. 2004, A\&A, 416, 853

Dubois, Y., Devriendt, J., Slyz, A., \& Teyssier, R. 2010, MNRAS, 409, 985

Dubois, Y., Gavazzi, R., Peirani, S., \& Silk, J. 2013, MNRAS, 433, 3297

Gabor, J. M., \& Davé, R. 2012, MNRAS, 427, 1816

Gabor, J. M., Davé, R., Oppenheimer, B. D., \& Finlator, K. 2011, MNRAS, 417, 2676

Gallazzi, A., Charlot, S., Brinchmann, J., White, S. D. M., \& Tremonti, C. A. 2005, MNRAS, 362, 41
Gerhard, O. E. 1981, MNRAS, 197, 179

Giovanelli, R., Haynes, M. P., Salzer, J. J., et al. 1994, AJ, 107, 2036

Graham, A. W., \& Worley, C. C. 2008, MNRAS, 388, 1708

Gunn, J. E., \& Gott, III, J. R. 1972, ApJ, 176, 1

Hernandez, X., \& Lee, W. H. 2004, MNRAS, 347, 1304

Hernquist, L. 1990, ApJ, 356, 359

Hernquist, L. 1992, ApJ, 400, 460

Hopkins, P. F., Cox, T. J., Kereš, D., \& Hernquist, L. 2008, ApJS, 175, 390

James, P. A., Shane, N. S., Beckman, J. E., et al. 2004, A\&A, 414, 23

Jiang, C. Y., Jing, Y. P., Faltenbacher, A., Lin, W. P., \& Li, C. 2008, ApJ, 675, 1095

Jonsson, P. 2006, MNRAS, 372, 2

Kannappan, S. J. 2004, ApJ, 611, L89

Kaviraj, S., Peirani, S., Khochfar, S., Silk, J., \& Kay, S. 2009, MNRAS, 394, 1713

Kennicutt, Jr., R. C. 1983, ApJ, 272, 54

Kennicutt, Jr., R. C. 1998, ApJ, 498, 541

Kennicutt, Jr., R. C., Tamblyn, P., \& Congdon, C. E. 1994, ApJ, 435, 22

Khochfar, S., \& Burkert, A. 2006, A\&A, 445, 403

Komatsu, E., Smith, K. M., Dunkley, J., et al. 2011, ApJS, 192, 18

Lee, J., \& Yi, S. K. 2013, ApJ, 766, 38

Li, C., Kauffmann, G., Heckman, T. M., Jing, Y. P., \& White, S. D. M. 2008, MNRAS, 385, 1903

Li, Y., Hernquist, L., Robertson, B., et al. 2007, ApJ, 665, 187

Lin, L., Koo, D. C., Willmer, C. N. A., et al. 2004, ApJ, 617, L9

Lotz, J. M., Jonsson, P., Cox, T. J., \& Primack, J. R. 2008, MNRAS, 391, 1137

Masters, K. L., Giovanelli, R., \& Haynes, M. P. 2003, AJ, 126, 158

Matthews, L. D., \& Wood, K. 2001, ApJ, 548, 150

McGaugh, S. S. 2005, ApJ, 632, 859

Merritt, D. 1996, AJ, 111, 2462

Mihos, J. C. 2004, in Recycling Intergalactic and Interstellar Matter, ed. P.-A. Duc, J. Braine, \& E. Brinks, IAU Symp., 217, 390

Mihos, J. C., \& Hernquist, L. 1994, ApJ, 437, 611

Mihos, J. C., \& Hernquist, L. 1996, ApJ, 464, 641

Moore, B., Katz, N., Lake, G., Dressler, A., \& Oemler, A. 1996, Nature, 379, 613

Naab, T., \& Burkert, A. 2003, ApJ, 597, 893

Negroponte, J., \& White, S. D. M. 1983, MNRAS, 205, 1009

Nickerson, S., Stinson, G., Couchman, H. M. P., Bailin, J., \& Wadsley, J. 2011, MNRAS, 415, 257

Patton, D. R., Carlberg, R. G., Marzke, R. O., et al. 2000, ApJ, 536, 153

Peirani, S., Hammer, F., Flores, H., Yang, Y., \& Athanassoula, E. 2009, A\&A, 496, 51

Peirani, S., Crockett, R. M., Geen, S., et al. 2010, MNRAS, 405, 2327

Pierini, D., Gordon, K. D., Witt, A. N., \& Madsen, G. J. 2004, ApJ, 617, 1022

Rocha, M., Jonsson, P., Primack, J. R., \& Cox, T. J. 2008, MNRAS, 383, 1281

Sanders, D. B., Soifer, B. T., Elias, J. H., et al. 1988, ApJ, 325, 74

Schawinski, K., Khochfar, S., Kaviraj, S., et al. 2006, Nature, 442, 888

Schawinski, K., Kaviraj, S., Khochfar, S., et al. 2007a, ApJS, 173, 512

Schawinski, K., Thomas, D., Sarzi, M., et al. 2007b, MNRAS, 382, 1415

Sheen, Y.-K., Yi, S. K., Ree, C. H., \& Lee, J. 2012, ApJS, 202, 8

Sijacki, D., Springel, V., Di Matteo, T., \& Hernquist, L. 2007, MNRAS, 380 877

Sijacki, D., Springel, V., \& Haehnelt, M. G. 2009, MNRAS, 400, 100

Silva, L., Granato, G. L., Bressan, A., \& Danese, L. 1998, ApJ, 509, 103

Springel, V. 2000, MNRAS, 312, 859

Springel, V. 2005, MNRAS, 364, 1105

Springel, V., Di Matteo, T., \& Hernquist, L. 2005a, ApJ, 620, L79

Springel, V., Di Matteo, T., \& Hernquist, L. 2005b, MNRAS, 361, 776

Stewart, K. R., Bullock, J. S., Wechsler, R. H., Maller, A. H., \& Zentner, A. R. 2008, ApJ, 683, 597

Teyssier, R., Moore, B., Martizzi, D., Dubois, Y., \& Mayer, L. 2011, MNRAS, 414, 195

Toomre, A. 1977, in Evolution of Galaxies and Stellar Populations, Proc. of the Conf. at Yale University, eds. B. M. Tinsley R. B. G. Larson \& D. Campbell, 401

Toomre, A., \& Toomre, J. 1972, ApJ, 178, 623

Tremaine, S., Gebhardt, K., Bender, R., et al. 2002, ApJ, 574, 740

Tuffs, R. J., Popescu, C. C., Völk, H. J., Kylafis, N. D., \& Dopita, M. A. 2004, A\&A, 419, 821

Tully, R. B., Pierce, M. J., Huang, J.-S., et al. 1998, AJ, 115, 2264

Valluri, M. 1993, ApJ, 408, 57

Wang, J., Hammer, F., Athanassoula, E., et al. 2012, A\&A, 538, A121

White, S. D. M., \& Rees, M. J. 1978, MNRAS, 183, 341

Xilouris, E. M., Byun, Y. I., Kylafis, N. D., Paleologou, E. V., \& Papamastorakis, J. 1999, A\&A, 344, 868

Yi, S. K., Lee, J., Jung, I., Ji, I., \& Sheen, Y.-K. 2013, A\&A, 554, A122

Zheng, X. Z., Bell, E. F., Somerville, R. S., et al. 2009, ApJ, 707, 1566 